\title{
Some latest achievements in immunology research
}

\author{
LIU Wei \\ Department of Biosciences and Nutrition, Karolinska Institute, NOVUM 14183, Huddinge, Sweden
}

Received September 15, 2011; accepted October 13, 2011

\begin{abstract}
Immunology has become as an attractive discipline of biomedical science in the 21 st century, integrated with the rapid development of molecular biology, cell biology, structural biology, genetics and other branches in life sciences. A special issue of $S c i$ China Life Sci was published in February 2010 to highlight recent progresses in immunology. Six review articles focusing on various specific topics were contributed by leading scientists who work in respective fields of immunology. Some progresses of immunology research in China are also described.
\end{abstract}

pattern recognition, $T$ cells, innate and adaptive immunity, costimulation, TRAF, plasmacytoid dendritic cells, humanized mouse model

Citation: Liu W. Some latest achievements in immunology research. Chinese Sci Bull, 2011, 56: 3890-3893, doi: 10.1007/s11434-011-4854-8

Immunity, the concept from immunis, a Latin word meaning "exempt", can be traced back to the plaque of Athens in 430 BC. Many other ancient nations have documented that people who had recovered from a previous bout of the disease would acquire mysterious ability to avoid contracting the illness for the second time. Early forms of vaccination were developed long before the word vaccination was first introduced by Edward Jenner in 1796 to specifically describe the injection of smallpox vaccine, but it was not until the 19th and 20th centuries before the concept developed into scientific theory [1]. In the 21 st century, immunology has become an indispensable branch of biomedical science that covers the study of all aspects of the immune system, and broadened its horizons with much research being performed in the more specialized niches, such as clinical immunology, developmental immunology, evolutionary immunology, cancer immunology and immunotherapy. Immunology deals with not only functioning of the immune system in health state, but malfunctions of the immune system in immunological disorders as well, thus deepening understanding of pathophysiology of human diseases and contributing to therapeutical and pharmaceutical developments.

To celebrate the 60th anniversary of Science China Press,

*Corresponding author (email: wei.liu.2005@gmail.com) a special issue of Sci China Life Sci summarizing recent advances in immunology came out in February 2010. Authors of the 6 review articles published on that issue, who are all leading immunologists, shared their understanding on different hot questions or focused on a single question of common interest from different views arisen from their respective expertise.

Vertebrates have evolved to protect themselves against infections by two different means: innate immunity and adaptive immunity, while invertebrates rely on innate immunity only. Both immune responses are tightly regulated in all organisms to prevent over-reaction and maintain immunological homeostasis [2-4]. In 1989, Janeway proposed a revolutionary concept that adaptive immunity is initiated by pattern recognition of innate immunity [5]. In a minireview article published on the special issue of immunology, Liu et al. [6] provided the first experimental evidence that microbial recognition by the innate immune system turns on the adaptive immune system and explained how the immune system controls the balance between sensing pathogenassociated molecular patterns (PAMPs) and the self-dangerassociated molecular patterns (DAMPs). Their previous work demonstrated the existence of costimulatory activity of bacterial components and the need of cis-presentation of both antigen and costimulartors for $\mathrm{T}$ cell activation $[7,8]$. 
The recent observation on the role of the CD24-SiglecG/10 pathway in repressing tissue injures indicates that this novel pathway very likely differentially regulate the recognition of DAMPs and PAMPs. Either SiglecG identified in mice or Siglec10 in human forms complexes with high mobility group protein-1 (HMGB1), an intracellular component able to induce inflammatory responses, through CD24, and negatively regulates DAMPs only in vivo.

Despite the protective role of the inflammatory response essential to the host, the response must be actively terminated after the acute phase. Failure to do so results in "bystander" damage to tissues and autoimmunity diseases. Recent studies have revealed that adaptive immune cells actively dampen initial innate response, as a mean of negative regulation on the inflammatory response. Tang et al. [9] summarized novel findings in this regard in another review article. It has become clear that conventional $\mathrm{T}$ cells not only activate innate cells to clear pathogens, but surprisingly take part in regulation of the innate immune response during the early phases of infection. There is an increasing body of evidence indicating that regulatory $\mathrm{T}$ cells (Treg), a subset of $\mathrm{CD}^{+} \mathrm{T}$ cells, can suppress functions of not only effector $\mathrm{T}$ cells but also innate cells [10-12]. A recent finding suggests an interesting feedback mechanism that allows $\mathrm{T}$ cells to selectively eliminate the bystander effect of the inflammatory response [13]. Both effector and memory $\mathrm{T}$ cells suppress potentially damaging inflammation through dampening the hyperactive inflammasome, yet leave the primary inflammatory response intact on the onset of immunity. The authors speculate that a feedback loop from the adaptive to the innate immune system was evolved in vertebrates whose innate inflammatory response might be insufficient to meet all the requirements.

The $\mathrm{T}$ cell pathways play a central role in regulating the immune response. Accumulating evidences indicate that the interaction of T-cell receptor (TCR) with antigenic peptide/major histocompatibility complex (MHC) alone is not sufficient to drive the activation of naive $\mathrm{T}$ cells leading to optimal immue responses [14]. A group of "costimulatory molecules" present on cell surface have been identified to actively suppress $\mathrm{T}$ cell responses in the presence of TCR signal [15]. Wang et al. [16], who discovered various costimulatory and coinhibitory molecules in $\mathrm{T}$ cell activation, reviewed the recent studies on two major families involved in $\mathrm{T}$ cell costimulation and regulation of adaptive immunity from perspective of structural immunology. The most extensively investigated receptors expressed on $\mathrm{T}$ cells and ligands expressed on the antigen-presenting cells or peripheral tissues include the members of the B7/CD28 and tumor-necrosis factor (TNF)/TNFR families. Molecular polymerization state and module organization of all these receptors and ligands are schematically represented in this review chapter, with detailed description of domain structures. Physiological function of each family member and therapeutic application of the B7-CD28/CTLA-4 and the
B7-H1/B7-DC/PD-1 pathways are well summarized as well. In the future, more structural information providing deeper insight into $\mathrm{T}$ cell costimulation will provide a sound basis for better studying the biological functions of the costimulatory pathways and encourage their application in therapy.

Tumor necrosis factor receptor-associated factors (TRAF) were initially discovered as adaptor proteins that couple the tumor necrosis factor receptor (TNFR) family to signaling pathways. More recently they have also been shown to be signal transducers of a large number of receptor families including the TNF receptor family and the Toll-like receptors-interleukin-1 receptors (TLR-IL-1R) family. Wang et al. [17] contributed to a minute review regarding the structure and function of the TRAF family. All the 6 members of the TRAF family share a C-terminal homology region termed the TRAF domain that is capable of binding to the cytoplasmic domain of receptors, and to other TRAF proteins. The 3D structures of the TRAF domain and another $\mathrm{N}$-terminal RING domain are detailedly described in this article. Research studies in the past few years have established the central role of the TRAF proteins in inflammation and immunity by regulating both canonical and noncanonical nuclear factor $\kappa \mathrm{B}(\mathrm{NF}-\kappa \mathrm{B})$ pathways as well as type 1 interferons production. Tremendous progresses have been made recently, for instance, the recruitment of TRAF $2 / 5$ in a signaling complex mediating the TNF-induced canonical $N F-\kappa B$ activation [18]; the oligomerization of TRAF6 to activate its E3 ubiquitin ligase activity, further leading to transforming growth factor $\beta$-activated kinase 1 (TAK1) and downstream signal transduction in the TLR-IL-1Rinduced canonical NF- $\kappa \mathrm{B}$ pathway [19]; and the degradation of TRAF2 and TRAF3 resulting in the stabilization and accumulation of NF- $\mathrm{BB}$-inducing kinase (NIK) in non-

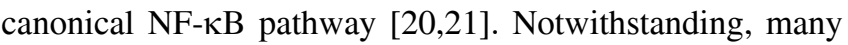
aspects about the roles played by the TRAF family in $\mathrm{NF}-\kappa \mathrm{B}$ remain unclear and require more investigations.

Dendritic cells (DCs) are professional antigen-presenting cells within the immune system, crucial for the innate and adaptive immune responses to infections. Plasmacytoid dendritic cells (pDCs), an important type of dendritic cells, represent a unique and crucial immune cell population capable of producing type I interferons (IFNs) in response to viral infection [22]. Tang et al. [23] working in the laboratory from which pDCs were indentified 10 year ago, dedicated another chapter by reviewing the remarkable progress within the past decade in understanding pDC biology, with focusing on the molecular mechanisms in antiviral immunity and autoimmunity [23]. Cellular properties, including the morphology, surface phenotype, development, location and migration of human pDCs are recounted in the first part, while physiological and pathophysiological functions of pDCs in both antiviral immunity and autoimmunity are narrated in the following part. Increasingly available data suggest that pDCs sense nucleic acids through TLR7 and TLR9, 
two members of the Toll-like receptor family [24,25], and link innate and adaptive immunity by promoting the antiviral functions of myeloid dendritic cells (mDCs), natural killer (NK) cells, T cells and B cells [26]. Considerable emerging evidences argue that the chronically activated pDCs and the IFN- $\alpha$ that they produce in response to self DNA/RNA are contributing factors in the pathogenesis of some autoimmune diseases, such as systemic lupus erythematosus (SLE) and psoriasis. Despite greatly enhanced understanding of pDC biology achieved recently, a number of fascinating questions requiring further investigation are crucial to develop safe and effective immunotherapy for viral infectious diseases and autoimmune diseases.

For better understanding human diseases without the added risk of causing harm to an actual human being during the process, modeling diseases on animals is an irreplaceable approach. Zhang et al. [27] reviewed the establishment of a new generation of humanized mice, an animal model for studying HIV-1 immuno-pathogenesis. They propose that the criteria of a robust animal model for "hypothesis-testing/mechanistic" research in human immunology and immuno-pathology should be: (1) it has well studied hemato-lymphoid organs and target cells similar to those of humans; (2) the human pathogens establish infection and lead to relevant diseases; and (3) it is genetically inbred and can be manipulated via genetic, immunological and pharmacological means. Unfortunately, some immunological findings in mice cannot translate directly to the human immune system and many human pathogens infect human cells only. Significant improvements have been achieved recently in humanized mouse models that to some extent overcome these limitations. Three mouse models commonly used in current research of human immune system and HIV-1 diseases are introduced in detail in this article, and novel mechanistic understanding gained from latest studies on HIV-1 infection and pathogenesis using NTB-hu HSC mice is summarized at length as well. Some featured events during clinical latency prior to AIDS developing, such as drop of both $\mathrm{CD}^{+}$and $\mathrm{CD}^{+} \mathrm{T}$ cells, depletion of $\mathrm{CCR}^{+} \mathrm{CD} 4^{+}$memory $\mathrm{T}$ cells in the gut-associated lymphoid tissue (GALT), and elevated/reduced level of regulatory $\mathrm{T}$ (Treg) cells in infectious diseases and autoimmune diseases respectively, have been able to be scrutinized using appropriate mouse models. In the future, it will be exciting to model directed human tissue-specific differentiation in humanized mice.

In the past decade, Chinese scientists working in domestic institutes have achieved fruitful outcome in immunity studies, coincident with the giant economic development occurring in China. More and more young talented immunologists have been attracted by lots of newly established laboratories. Longing for comprehensive and deeper mechanistic understanding of human pathology form irresistible impetus; revolutionary advances of biotechnology, e.g. gene knockout and RNAi knockdown, provide significant tech- nical support; and increasing funding resources in China establish solid material basis for current immunology research. Noteworthy contributions made by Chinese laboratories in 2009 and 2010 include monoclonal antibodies production [28-31], studies on antigen-antibody binding site [32,33], Toll-like receptor (TLR) mediated signaling pathways [34,35], inflammasome and inflammatory response [36-38], and HIV-1 infection [39,40]. Owing to constant and steady increase of funding resources as well as progressively improved research conditions, more significant achievements generated in domestic laboratories in China are highly expected.

1 Cheng G, Liu Y, Tang H. Vaccination is one of the most significant inventions in medical science. Sci China Life Sci, 2010, 53: 157-158

2 Aggarwal K, Silverman N. Positive and negative regulation of the Drosophila immune response. BMB Rep, 2008, 41: 267-277

3 Liew F Y, Xu D, Brint E K, et al. Negative regulation of toll-like receptor-mediated immune responses. Nat Rev Immunol, 2005, 5: 446-458

4 Meng F Y, Mai K S, Ma H M, et al. The evolution of echinoderm immunology. Prog Biochem Biophys, 2009, 36: 803-809

5 Janeway C A Jr. Approaching the asymptote? Evolution and revolution in immunology. Cold Spring Harb Symp Quant Biol, 1989, 54: $1-13$

6 Liu Y, Chen G, Zheng P, et al. On self-nonself discrimination in pattern recognition. Sci China Life Sci, 2010, 53: 169-171

7 Liu Y, Janeway C A Jr. Microbial induction of co-stimulatory activity for CD4 T-cell growth. Int Immunol, 1991, 3: 323-332

8 Liu Y, Janeway C A Jr. Cells that present both specific ligand and costimulatory activity are the most efficient inducers of clonal expansion of normal CD4 T cells. Proc Natl Acad Sci USA, 1992, 89: 3845-3849

9 Tang $\mathrm{H}, \mathrm{Fu} \mathrm{Y}$. A new role for T cells in dampening innate inflammatory responses. Sci China Life Sci, 2010, 53: 190-194

10 Maloy K J, Salaun L, Cahill R, et al. CD4 $4^{+} \mathrm{CD} 25^{+} \mathrm{T}(\mathrm{R})$ cells suppress innate immune pathology through cytokine-dependent mechanisms. J Exp Med, 2003, 197: 111-119

11 Smyth M J, Teng M W, Swann J, et al. CD4 ${ }^{+} \mathrm{CD} 25^{+} \mathrm{T}$ regulatory cells suppress NK cell-mediated immunotherapy of cancer. J Immunol, 2006, 176: 1582-1587

12 Tiemessen M M, Jagger A L, Evans $\mathrm{H} \mathrm{G}$, et al. CD $4^{+} \mathrm{CD} 25^{+} \mathrm{Foxp}^{+}$ regulatory $\mathrm{T}$ cells induce alternative activation of human monocytes/ macrophages. Proc Natl Acad Sci USA, 2007, 104: 19446-19451

13 Guarda G, Dostert C, Staehli F, et al. T cells dampen innate immune responses through inhibition of NLRP1 and NLRP3 inflammasomes. Nature, 2009, 460: 269-273

14 Lenschow D J, Walunas T L, Bluestone J A. CD28/B7 system of T cell costimulation. Annu Rev Immunol, 1996, 14: 233-258

15 Chen L. Co-inhibitory molecules of the B7-CD28 family in the control of T-cell immunity. Nat Rev Immunol, 2004, 4: 336-347

16 Wang S, Chen L. Structural immunology of costimualtory and coinhibitory molecules. Sci China Life Sci, 2010, 53: 183-189

17 Wang Y, Zhang P, Liu Y, et al. TRAF-mediated regulation of immune and inflammatory responses. Sci China Life Sci, 2010, 53: 159-168

18 Micheau O, Tschopp J. Induction of TNF receptor I-mediated apoptosis via two sequential signaling complexes. Cell, 2003, 114: $181-190$

19 Cao Z, Xiong J, Takeuchi M, et al. TRAF6 is a signal transducer for interleukin-1. Nature, 1996, 383: 443-446

20 Annunziata C M, Davis R E, Demchenko Y, et al. Frequent engagement of the classical and alternative NF-kappaB pathways by diverse genetic abnormalities in multiple myeloma. Cancer Cell, 2007, 12: 115-130 
21 Keats J J, Fonseca R, Chesi M, et al. Promiscuous mutations activate the noncanonical NF-kappaB pathway in multiple myeloma. Cancer Cell, 2007, 12: 131-144

22 Siegal F P, Kadowaki N, Shodell M, et al. The nature of the principal type 1 interferon-producing cells in human blood. Science, 1999, 284: $1835-1837$

23 Tang F, Du Q, Liu Y J. Plasmacytoid dendritic cells in antiviral immunity and autoimmunity. Sci China Life Sci, 2010, 53: 172-182

24 Diebold S S, Kaisho T, Hemmi H, et al. Innate antiviral responses by means of TLR7-mediated recognition of single-stranded RNA. Science, 2004, 303: 1529-1531

25 Krug A, French A R, Barchet W, et al. TLR9-dependent recognition of MCMV by IPC and DC generates coordinated cytokine responses that activate antiviral NK cell function. Immunity, 2004, 21: 107-119

26 Ito T, Amakawa R, Inaba M, et al. Differential regulation of human blood dendritic cell subsets by IFNs. J Immunol, 2001, 166: 2961-2969

27 Zhang L G, Meissner E, Chen J Z, et al. Current humanized mouse models for studying human immunology and HIV-1 immuno-pathogenesis. Sci China Life Sci, 2010, 53: 195-203

28 Luo R J, Siriguleng, Liu X H, et al. Preparation and immunological identification of monoclonal antibody against JSRV-CA. Prog Biochem Biophys, 2009, 36: 108-114

29 Qu J, Zhang H, Zhang L Y, et al. Development of anti-CD44 therapeutic antibody for acute myeloid leukemia. Prog Biochem Biophys, 2009, 36: 190-197

30 Guo Z F, He S Y, Zhu B Y, et al. Preparation of Anti-hTM monoclonal antibody by using hTM expression cell line. Prog Biochem Biophys, 2009, 36: 441-447
31 Pei X H, Chi S, Wang J T, et al. Generation and application of active recombinant mouse tissue factor and its function-blocking monoclonal antibody. Prog Biochem Biophys, 2009, 36: 601-607

32 Jia J Y, Zhou H Z, Tang J. The study of mouse TNF- $\alpha$ functional domain and its neutralizing antibody binding site. Prog Biochem Biophys, 2009, 36: 424-430

33 Jia J Y, Wang Y B, Tang J. A new strategy for epitope mapping by yeast surface display system. Prog Biochem Biophys, 2009, 36: 305-310

34 Sun B, Han D S. Negative regulation of Toll-like receptors signaling pathways. Prog Biochem Biophys, 2009, 36: 1516-1522

35 Zhong T Y, Tang J, Chen D Y, et al. Using FRET to study the interaction domain of TLR4 binding to MD-2 in living cells. Prog Biochem Biophys, 2009, 36: 1451-1457

36 Zhu X M, Yao Y M, Sheng Z Y. Inflammasome and inflammatory response. Prog Biochem Biophys, 2010, 37: 129-137

37 Sun L, Liu D B, Yang Y D, et al. Regulation of antiviral innate immune responses by human coronavirus. Prog Biochem Biophys, 2010, 37 : 239-244

38 Li Z Y, Wu Y M, Huang Q L, et al. Localization and characterization of hypothetical protein CT358 in the Chlamydia Trachomatisinfected cells. Prog Biochem Biophys, 2009, 36: 549-555

39 An Q X, Lei Y F, Yang J, et al. Inhibition of HIV-1 infection by CCR5Delta32 protein expressed in human PBMCs. Prog Biochem Biophys, 2009, 36: 1141-1145

40 He H Q, Hu J P, Liu B, et al. Solubility comparison and molecular dyanmics simulation analysis of wild type and F185K mutant type HIV-1 integrase catalytic domain. Prog Biochem Biophys, 2009, 36 : 1146-1153

Open Access This article is distributed under the terms of the Creative Commons Attribution License which permits any use, distribution, and reproduction in any medium, provided the original author(s) and source are credited. 